\title{
EFFECT OF SINGLE AND MULTIPLE PASSIVE STRETCHING EXERCISES UPON HEART RATE VARIABILITY IN INDIVIDUALS WITH HIGH AND LOW FLEXIBILITY LEVELS
}

original paper

( ) University School of Physical Education in Wroclaw

DOI: https://doi.org/10.5114/hm.2021.100320

ERCOLE DA CRUZ RUBINI ${ }^{1,2}$, PAULO DE TARSO VERAS FARINATTI ${ }^{3,4}$, CAMILA CARNEIRO DIANO ${ }^{1}$, MATHEUS JOSÉ PASSOS BENTO VIANNA DE OLIVEIRA ${ }^{1}$, ELIREZ BEZERRA DA SILVA ${ }^{2}$

${ }^{1}$ Laboratory of Exercise Physiology, Estácio de Sá University, Rio de Janeiro, Brazil

${ }^{2}$ Research Group on Exercise and Health Science, Rio de Janeiro State University, Rio de Janeiro, Brazil

${ }^{3}$ Laboratory of Physical Activity and Health Promotion, Rio de Janeiro State University, Rio de Janeiro, Brazil

${ }^{4}$ Sciences of Physical Activity Graduate Program, Salgado de Oliveira University, Niterói, Brazil

\section{ABSTRACT}

Purpose. This study investigated heart rate variability indices within acute passive stretching sessions with different exercise amounts in individuals with low and high flexibility.

Methods. Fifty healthy men (23 \pm 3 years) were randomly assigned into 4 subgroups: low flexibility-low amount, low flexibility-high amount, high flexibility-low amount, high flexibility-high amount. In groups with low amount of exercises, a single stretching of hamstrings to the maximal range of motion was performed; in high amount sessions, 6 exercises for lower and upper limbs were applied. In both protocols, individuals performed two 30-s sets using the passive static method. Heart rate variability indices in time and frequency domains were calculated from beat-to-beat intervals 15 min before, during the stretching, and along 30-min after exercise.

Results. Multifactorial ANOVA showed that in all cases, passive stretching induced parasympathetic suppression and sympathetic increase vs. baseline $(p<0.05)$. After exercise, interaction between repeated measures was significant only for exercise amount. An increase vs. baseline occurred in high frequency power ( $\triangle \%$ low amount: $6.7 \%$, high amount: $-9.7 \%$; $p<0.05)$ and root mean square of successive differences ( $\Delta \%$ low amount: $17.1 \%$, high amount: $14.1 \% ; p<0.05)$. The low frequency power ( $\Delta \%$ low amount: $-4.2 \%$, high amount: $-6.9 \% ; p<0.05)$ and low-to-high frequency power ratio $(\Delta \%$ high amount: $-6.7 \% ; p<0.05)$ decreased.

Conclusions. Sympathetic modulation increased during acute stretching. Vagal increase and sympathetic reduction occurred during recovery, irrespective of flexibility. These changes were shorter after sessions including single (20 min) vs. multiple (30 min) exercises.

Key words: heart rate, range of motion, autonomic modulation, physical exercise

\section{Introduction}

The preservation of muscle strength and flexibility is recommended within health-oriented physical exercise programmes [1]. For flexibility training, stretching exercises [2] are used. Prior studies have investigated the potential cardiovascular effects of this type of exercise, including changes in heart rate variability (HRV) [3-8]. HRV has been used to assess the autonomic modulation after a variety of physical exercises [9-11]. It is known that physical exercises can positively influence the autonomic nervous system, reducing sympathetic activity and increasing parasympathetic activity $[12,13]$. Greater parasympathetic outflow speeds heart rate recovery after exercise, which seems to be associated with a lower risk of cardiovascular diseases [14-16]. In this context, research on HRV responses to different types of exercise might be relevant for a better prescription of physical training designed to improve cardiovascular health.

Correspondence address: Ercole da Cruz Rubini, Laboratory of Exercise Physiology, Estácio de Sá University, Rua André Rocha, 838, Taquara, Rio de Janeiro, RJ, Brazil, zip code: 22710-560, e-mail: ercolerubini@yahoo.com.br

Received: April 16, 2019

Accepted for publication: May 16, 2020

Citation: da Cruz Rubini E, de Tarso Veras Farinatti P, Diano CC, de Oliveira MJPBV, da Silva EB. Effect of single and multiple passive stretching exercises upon heart rate variability in individuals with high and low flexibility levels. Hum Mov. 2021;22(3):11-18; doi: https://doi.org/10.5114/hm.2021.100320. 
Although there are few studies investigating the effects of stretching on cardiac autonomic control, some evidence suggests that favourable alterations may occur owing to the activation of mechanoreceptors during isometric contractions typically performed [17, 18]. Stretching is related to static contractions of both agonist and antagonist (muscle spindle reflex) muscles because of the stimulation of mechanoreceptors influencing autonomic and haemodynamic outcomes [3, 17]. This appears to be especially true in subjects with low flexibility levels, at least with regard to acute HRV responses. In fact, of the 6 studies that previously investigated the effects of muscle stretching on HRV [3-8], only 1 enrolled participants with good flexibility levels [6]. Coincidentally, this was 1 of the 2 experiments failing to observe changes in HRV [6]; the other trial did not describe the participants' flexibility [7]. The remaining studies involved individuals classified as having low flexibility levels [3] or pregnant women (flexibility not reported) [4], observing increased HRV [3, 4]. We found only 2 studies investigating the chronic effects of flexibility training on cardiac autonomic control, which reported greater parasympathetic modulation after training in bodybuilders with limited flexibility [5] and obese postmenopausal women [8].

On the other hand, the analysis of data from these few studies gives room to think that besides the individual flexibility level, the amount of stretching can also influence HRV responses. In fact, the trial applying the lowest amount of exercises ( 2 sets of pectoral muscles stretching, total duration $<2 \mathrm{~min}$ ) did not report significant changes in HRV [6]. In turn, trials that applied procedures involving 3 sets of multiple exercises with total duration of approximately 10-20 min detected an HRV increase [3, 4]. The amount of exercises applied in the 2 chronic studies can also be considered high - bodybuilders performed 15 min of stretching exercises for large muscle groups [5], while obese postmenopausal women underwent 38 stretching exercises within 50-min training sessions [8].

In short, if the intensity of isometric contractions within stretching exercises tends to be greater when individuals have low flexibility, a greater amount of exercises may also increase the mechanical stimuli of mechanoreceptors, therefore influencing the autonomic outflow. However, to date, no study has investigated the relative role of these factors to produce autonomic changes.

Thus, the present study explored the effects of acute flexibility training performed with different amounts of exercises upon the cardiac autonomic modulation assessed by HRV, in young men classified as exhibit- ing low and high flexibility levels. We hypothesized that: (a) individuals with low flexibility would be more susceptible to exhibit autonomic changes vs. those with high flexibility; (b) multiple sets of varied exercises for large muscle groups would be more likely to influence HRV than a single-exercise protocol focusing on a small muscle group.

\section{Material and methods}

\section{Participants}

This transversal descriptive study involved 50 healthy young males aged $18-30$ years ( $23 \pm 3$ years, $75.8 \pm$ $12.7 \mathrm{~kg}, 1.75 \pm 0.06 \mathrm{~m}$ ), recruited by means of advertising through posters and social networks. The following exclusion criteria were adopted: (a) chronic pain or injury to the joints and muscles engaged in the tests and exercises proposed; (b) smoking; and (c) use of drugs with effects on the cardiovascular responses or autonomic system, at rest or during exercise.

\section{Experimental design}

The experiment was carried out during 2 visits. On the first visit, the participants completed a questionnaire covering personal information, injury history, and habitual physical activity. In addition, they underwent anthropometric and flexibility measurements. On the basis of this initial flexibility assessment, the subjects were classified as exhibiting low flexibility or high flexibility; then, they were randomly assigned into groups performing a low (LA) or high (HA) amount of exercises. They were instructed not to take any strenuous physical exercise and abstain from alcohol, coffee, and tea in the $24 \mathrm{~h}$ preceding the tests and experimental sessions, while the last meal should be light and consumed at least $3 \mathrm{~h}$ before.

On the second visit, passive stretching exercises were performed to the maximal range of motion, in accordance with the prior randomization (LA and HA). LA exercise sessions consisted of 2 sets (30 s each) of a single passive static stretching exercise involving a large muscle group (hamstrings). HA exercise sessions included 2 sets (also $30 \mathrm{~s}$ each) of 6 passive static stretching exercises, also performed to the maximal range, involving large muscle groups (hamstrings, adductors, piriformis, quadriceps, trunk, and pectoral muscles). In both groups, 15-s intervals between sets were allowed. All exercise sessions were conducted by experienced instructors blinded for the purposes of the study. After the acute stretching sessions, the partici- 
pants resumed the supine position and remained at rest for $30 \mathrm{~min}$, during which beat-to-beat heart rate intervals were assessed with a cardiotachometer. All visits were performed between 14:00 and 18:00 $\mathrm{h}$ in a quiet environment, to reduce the potential influence of HRV circadian fluctuations. The temperature was kept at $21-23^{\circ} \mathrm{C}$ and the subjects were instructed to maintain their usual breathing pattern during the assessments.

\section{Procedures}

\section{Flexibility assessment}

The flexibility measurements were performed blindly by an independent evaluator, with the Flexitest $[19,20]$. In this method, the maximum passive physiological flexibility is measured in 20 movements in the joint groups of the ankle, knee, hip, trunk, elbow, wrist, and shoulder, with scores ranging from 0 to 4 points. The sum of the results for each movement produces a general flexibility index called the Flexindex, which varies from 0 to 80 .

\section{Heart rate variability assessment}

Beat-to-beat heart rate intervals were measured to calculate RR intervals, with the participants in the supine position, with the use of a Polar V800 cardiotachometer (Polar Electro Oy ${ }^{\mathrm{TM}}$, Kempele, Finland). Measurements were taken in 3 situations: $15 \mathrm{~min}$ at rest (before exercise); during the stretching exercise sessions; and along the 30-min postexercise recovery (every $10 \mathrm{~min}$ ). The RR interval time series were transferred to the Kubios HRV Analysis ${ }^{\mathrm{TM}}$ version 2.0 software
(Biosignal Analysis and Medical Imaging Group ${ }^{\mathrm{TM}}$, Kuopio, Finland). The power spectral density was estimated with the nonparametric method of the fast Fourier transform, after detrending the time series with the a priori smoothing method and decimation in frequency of $4 \mathrm{~Hz}$, with cubic spline interpolation. The following HRV indices were calculated in the time domain: standard deviation of all normal RR intervals recorded in one-time interval (SDNN) and root mean square of the successive differences (RMSSD), both related to parasympathetic activity [21]. In the frequency domain, the following indices were obtained: low frequency power (LF: 0.04-0.15 Hz), with sympathetic predominance [21]; high frequency power (HF: $0.15-0.40 \mathrm{~Hz}$ ), with parasympathetic predominance [21]; ratio between LF and HF (LF/HF), usually adopted as reflecting the sympathovagal balance [21]. All indices in the frequency domain were expressed in normalized units (n.u.).

\section{Stretching exercise sessions}

Participants with low and high flexibility levels were assigned into subgroups performing different amounts of stretching exercises. As abovementioned, the individuals assigned to LA performed a single stretching exercise for the hamstrings (position 1, Figure 1). Those in the HA subgroup performed 6 exercises involving the hamstrings, adductor, piriformis, quadriceps, trunk, and pectoral muscles (positions 1-6, Figure 1). In all cases, 2 sets of each exercise were performed to the maximal range of motion by means of the static passive method, using the point of muscle discomfort as load reference. The maximum extension positions were always maintained for $30 \mathrm{~s}$, interspersed with 15-s

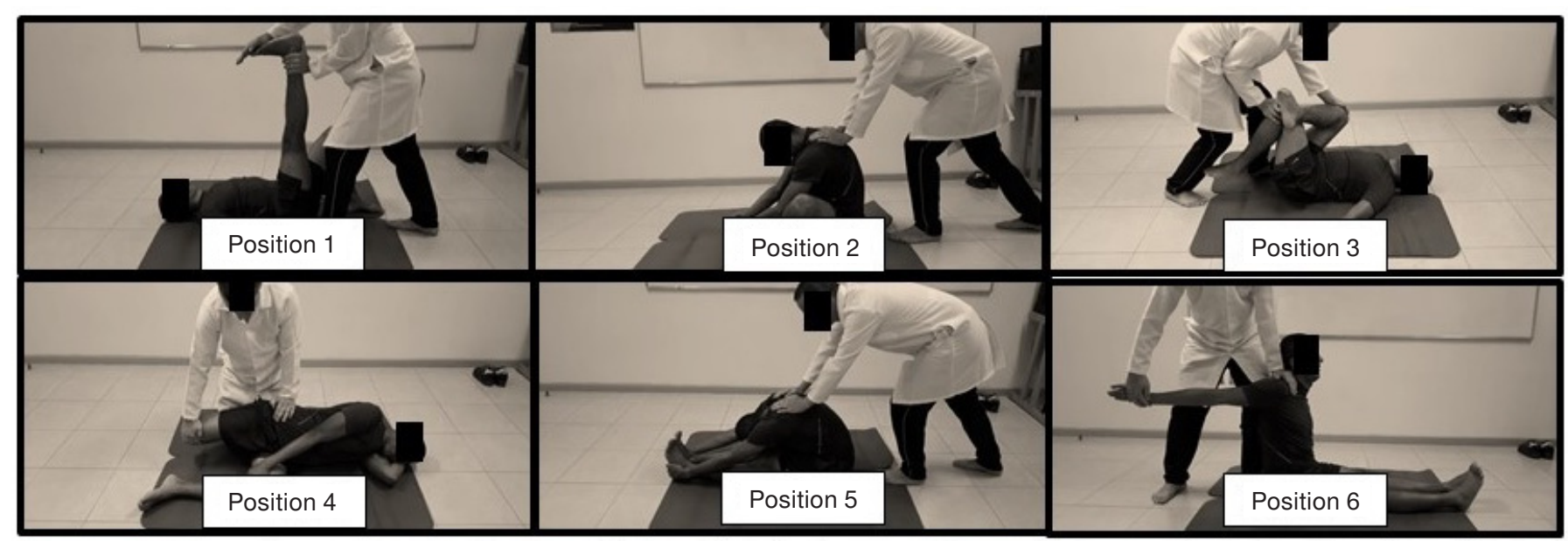

Figure 1. Six passive static stretching exercises applied in the low amount (position 1) and high amount (positions 1-6) procedures 
intervals between unilateral or bilateral sets, as well as between exercises.

\section{Statistics}

A prior sample calculation was made with the consideration of the power of 0.80 for 5 repeated measures, 4 experimental groups, and $\alpha$ of 0.05 . In addition, the effect size (Cohen's $f$ ) was set at 0.10 , correlation between repeated measures at 0.90 , and $\varepsilon$ at 1.0 . The $\mathrm{G}^{*}$ Power $^{\mathrm{TM}}$ version 3.1.7 software (University of Kiel, Kiel, Germany) was used. A sample size of 40 individuals was estimated as necessary. In accordance with the classification proposed by the Flexitest, individuals who obtained up to 40 points were considered as presenting low flexibility $(n=25$; Flexindex: $33.5 \pm$ 5.1 ), while those who scored 41 points or above were allocated to the high flexibility group $(n=25$; Flexindex: $48.0 \pm 4.5)$.

The normality and homoscedasticity/sphericity of data were confirmed by the Kolmogorov-Smirnov and Levene's tests, respectively. Therefore, data are presented as mean \pm standard deviation. In order to test possible differences between HRV indices across the experimental groups, a 3-way ANOVA with repeated measures $(2 \times 2 \times 5)$ was used (before exercise, during the stretching sessions, and 10, 20, 30 min after exercise). In all cases, Tukey's post-hoc verifications were applied in the event of significant $F$-ratios, with the significance level fixed at $\alpha=0.05$. Since the flexibility level presented no interaction with the repeated measures, further analysis neutralizing for this factor was made. Calculations were performed with the Statistica 7.0 software (StatSoft ${ }^{\mathrm{TM}}$ Inc., Tulsa, USA). Finally, the actual statistical power of the performed comparisons was calculated on the basis of effect sizes (Cohen's $f$ ) and correlations between repeated measurements, with the $G^{*}$ Power version 3.1.7 software (University of Kiel, Kiel, Germany).

\section{Ethical approval}

The research related to human use has complied with all the relevant national regulations and institutional policies, has followed the tenets of the Declaration of Helsinki, and has been approved by the Research Ethics Committee of the Estácio de Sá University (CAAE 65584417.0.0000.5284).

\section{Informed consent}

Informed consent has been obtained from all individuals included in this study.

\section{Results}

All participants completed the experiment and there was no report of discomforts related to the stretching exercise protocols. Table 1 presents the mean \pm standard deviation values for SDNN, RMSSD, HF, LF, and $\mathrm{LF} / \mathrm{HF}$ in the experimental groups. A significant interaction was detected for SDNN, RMSSD, HF, LF, and $\mathrm{LF} / \mathrm{HF}$ between sessions performed with different amounts of exercises. There was no interaction between HRV indices vs. isolate flexibility level or combination between the flexibility level and amount of exercises.

Table 2 presents mean \pm standard deviation values for SDNN, RMSSD, HF, LF and LF/HF in groups that performed LA and HA of exercises, after neutralizing the flexibility factor, which did not interact with the repeated measures. Sympathetic predominance in the stretching exercise sessions was confirmed and followed by increased parasympathetic and lowered sympathetic outflows after exercise. The sympathetic withdrawal seemed to be longer after HA (30 min) than LA (20 $\mathrm{min})$ sessions.

Table 3 shows the post-hoc statistical power obtained for HRV indices, based on the actual correlation between repeated measures and Cohen's $f$ effect size. With the number of participants in the study $(n=50)$, the statistical power was $\geq 0.99$. This indicates that differences in HRV indices were not obtained by chance, confirming the hypothesis that the amount of stretching exercises influenced these markers of cardiac autonomic modulation.

\section{Discussion}

Our findings suggest that a single session of passive stretching exercises performed to the maximal range of motion was capable to induce changes in HRV outcomes, regardless of the individual's flexibility level. Overall, during the exercise sessions, there was an increase in sympathetic and decrease in parasympathetic modulation. Along postexercise recovery, the vagal modulation increased, while indices predominantly reflecting the sympathetic modulation decreased. Changes in the sympathovagal balance were longer after sessions including HA vs. LA of stretching exercises.

The hypothesis that the autonomic control would be more responsible in individuals with low vs. high flexibility was derived from the results of 4 prior available studies [3, 4, 6, 7]. Two trials [3, 4] included subjects with low flexibility and reported a significant impact of static stretching on HRV. On the contrary, the study performed with individuals classified as exhibit- 
Table 1. Mean \pm standard deviation values for SDNN, RMSSD, HF, LF, and LF/HF in groups with low flexibility and low amount of exercises (LFLA), low flexibility and high amount of exercises (LFHA), high flexibility and low amount of exercises (HFLA), and high flexibility and high amount of exercises (HFHA) $(n=50)$

\begin{tabular}{|c|c|c|c|c|c|}
\hline \multirow[b]{2}{*}{ HRV index } & \multirow[b]{2}{*}{ Moment } & \multicolumn{4}{|c|}{ Group } \\
\hline & & $\begin{array}{c}\text { LFLA } \\
(n=12)\end{array}$ & $\begin{array}{l}\text { LFHA } \\
(n=13)\end{array}$ & $\begin{array}{l}\text { HFLA } \\
(n=12)\end{array}$ & $\begin{array}{l}\text { HFHA } \\
(n=13)\end{array}$ \\
\hline \multirow{5}{*}{ SDNN (ms) } & Before exercise & $55.76 \pm 20.67$ & $47.4 \pm 20.3$ & $56.2 \pm 22.5$ & $45.4 \pm 19.1$ \\
\hline & During exercise & $42.81 \pm 18.94^{t}$ & $53.4 \pm 13.9^{t}$ & $51.4 \pm 17.6^{\mathrm{t}}$ & $55.7 \pm 16.0^{\mathrm{t}}$ \\
\hline & 10 min after exercise & $60.31 \pm 25.77^{\mathrm{w}}$ & $52.2 \pm 24.6$ & $60.5 \pm 21.8^{w}$ & $43.4 \pm 14.5$ \\
\hline & 20 min after exercise & $65.93 \pm 28.18^{*}$ & $53.7 \pm 22.9$ & $64.7 \pm 25.5^{*}$ & $47.4 \pm 18.6$ \\
\hline & 30 min after exercise & $64.13 \pm 24.38^{\phi}$ & $53.4 \pm 18.8$ & $69.6 \pm 27.0^{\phi}$ & $53.8 \pm 25.5$ \\
\hline \multirow{5}{*}{ RMSSD (ms) } & Before exercise & $54.4 \pm 23.5$ & $46.2 \pm 24.4$ & $65.9 \pm 36.4$ & $47.4 \pm 24.1$ \\
\hline & During exercise & $36.0 \pm 18.5^{\mathrm{t}}$ & $38.4 \pm 17.5^{\mathrm{t}}$ & $45.3 \pm 21.3^{t}$ & $40.2 \pm 15.3^{t}$ \\
\hline & 10 min after exercise & $62.5 \pm 27.8^{w}$ & $55.2 \pm 32.4^{\mathrm{w}}$ & $70.2 \pm 33.6^{\mathrm{w}}$ & $47.3 \pm 20.0^{w}$ \\
\hline & 20 min after exercise & $67.2 \pm 30.0^{*}$ & $55.4 \pm 31.9 *$ & $73.6 \pm 36.7^{*}$ & $51.4 \pm 24.7^{*}$ \\
\hline & 30 min after exercise & $63.2 \pm 25.0^{\phi}$ & $53.2 \pm 27.4^{\phi}$ & $77.1 \pm 36.2^{\phi}$ & $57.3 \pm 33.0^{\phi}$ \\
\hline \multirow{5}{*}{ HF (n.u.) } & Before exercise & $39.2 \pm 12.7$ & $40.7 \pm 15.7$ & $50.3 \pm 11.3$ & $42.0 \pm 10.8$ \\
\hline & During exercise & $30.3 \pm 11.6^{\mathrm{t}}$ & $21.5 \pm 12.9^{t}$ & $33.7 \pm 13.0^{t}$ & $19.6 \pm 5.3^{t}$ \\
\hline & 10 min after exercise & $44.1 \pm 14.8^{\mathrm{w}}$ & $45.8 \pm 15.4^{\mathrm{w}}$ & $51.5 \pm 13.3^{w}$ & $43.1 \pm 13.8^{w}$ \\
\hline & 20 min after exercise & $42.8 \pm 15.9 *$ & $45.9 \pm 19.6^{*}$ & $49.7 \pm 12.6^{*}$ & $44.9 \pm 15.2^{*}$ \\
\hline & 30 min after exercise & $37.6 \pm 14.1^{\phi}$ & $42.5 \pm 18.3^{\phi}$ & $45.1 \pm 11.8^{\phi}$ & $39.4 \pm 10.7^{\phi}$ \\
\hline \multirow{5}{*}{ LF (n.u.) } & Before exercise & $53.9 \pm 11.5$ & $54.7 \pm 15.1$ & $46.1 \pm 10.5$ & $51.9 \pm 8.7$ \\
\hline & During exercise & $62.2 \pm 11.2^{\mathrm{t}}$ & $66.3 \pm 10.0^{\mathrm{t}}$ & $54.5 \pm 11.2^{\mathrm{t}}$ & $67.3 \pm 5.1^{\mathrm{t}}$ \\
\hline & 10 min after exercise & $51.3 \pm 14.6^{\mathrm{w}}$ & $50.0 \pm 15.2^{\mathrm{w}}$ & $44.6 \pm 12.3^{\mathrm{w}}$ & $50.3 \pm 12.5^{\mathrm{w}}$ \\
\hline & 20 min after exercise & $51.6 \pm 15.8^{*}$ & $49.8 \pm 18.7^{*}$ & $46.4 \pm 11.6^{*}$ & $49.4 \pm 12.8^{*}$ \\
\hline & 30 min after exercise & $55.7 \pm 14.5$ & $49.2 \pm 15.7^{\phi}$ & $50.4 \pm 12.2$ & $52.9 \pm 8.6^{\phi}$ \\
\hline \multirow{5}{*}{$\mathrm{LF} / \mathrm{HF}$} & Before exercise & $1.6 \pm 0.7$ & $1.7 \pm 0.9$ & $1.0 \pm 0.4$ & $1.4 \pm 0.8$ \\
\hline & During exercise & $2.6 \pm 1.7^{\mathrm{t}}$ & $5.0 \pm 4.1^{\mathrm{t}}$ & $2.0 \pm 1.3^{t}$ & $3.8 \pm 1.4^{\mathrm{t}}$ \\
\hline & 10 min after exercise & $1.4 \pm 0.9^{w}$ & $1.3 \pm 1.0^{\mathrm{w}}$ & $1.0 \pm 0.6^{w}$ & $1.5 \pm 1.1^{w}$ \\
\hline & 20 min after exercise & $1.5 \pm 0.9^{*}$ & $1.6 \pm 1.5^{*}$ & $1.1 \pm 0.6^{*}$ & $1.3 \pm 0.6^{*}$ \\
\hline & 30 min after exercise & $2.0 \pm 1.7$ & $1.7 \pm 1.6^{\phi}$ & $1.3 \pm 0.8$ & $1.5 \pm 0.6^{\phi}$ \\
\hline
\end{tabular}

HRV - heart rate variability, SDNN - standard deviation of all normal RR intervals recorded in one-time interval, RMSSD - root mean square of the successive differences, HF - high frequency power, LF - low frequency power, LF/LH - low-to-high frequency power ratio, n.u. - normalized units ${ }^{\mathrm{t}} p<0.05$ for before exercise vs. during exercise, ${ }^{\mathrm{w}} p<0.05$ for during exercise vs. $10 \mathrm{~min}$ after exercise, ${ }^{*} p<0.05$ for during exercise vs. 20 min after exercise, ${ }^{\phi} p<0.05$ for during exercise vs. 30 min after exercise

ing good flexibility failed to detect autonomic changes [6]. The fourth study did not classify the participants with regard to their flexibility level [7].

It can be speculated that the rejection of the hypothesis stating that the flexibility level would influence HRV after acute stretching sessions may be due to the small difference $(p=0.376)$ between groups classified as presenting low flexibility (Flexindex: $33.5 \pm 5.1$ points) or high flexibility (Flexindex: $48.0 \pm 4.5$ points). Another explanation could be that since the passive static stretching used external force do reach the maximal range of motion, only slight isometric contractions of antagonistic muscles occurred during the exercises. Thus, the stimulation via mechanoreceptors would be lower in comparison with active static stretching [17]. In fact, previous studies reported that in the absence of voluntary muscle activation, there would be insufficient activation of metaboreceptors or mechanoreceptors to induce relevant haemodynamic changes via afferent stimulation [16, 21].

The total amount of stretching exercises in the 4 available studies $[3,4,6,7]$ was also discrepant. The 3 experiments that detected acute effects on HRV involved relatively long stretching sessions (10-20 min) $[3,4,7]$, while the trial failing to observe autonomic changes [6] applied 2 sets of pectoral stretching during only $30 \mathrm{~s}$ (total duration of $2 \mathrm{~min}$ ). Considering the present results, one could presume that the amount 
E. Rubini et al., Stretching and heart rate variability

Table 2. Mean \pm standard deviation values of SDNN, RMSSD, HF, LF, and LF/HF depending on the amount of exercises only $(n=50)$

\begin{tabular}{|c|c|c|c|}
\hline \multirow[b]{2}{*}{ HRV index } & \multirow[b]{2}{*}{ Moment } & \multicolumn{2}{|c|}{ Group } \\
\hline & & $\begin{array}{l}\text { Low amount } \\
\qquad(n=24)\end{array}$ & $\begin{array}{l}\text { High amount } \\
\qquad(n=26)\end{array}$ \\
\hline \multirow{5}{*}{ SDNN (ms) } & Before exercise & $56.0 \pm 4.1$ & $46.4 \pm 3.9$ \\
\hline & During exercise & $47.1 \pm 3.3$ & $54.5 \pm 3.2$ \\
\hline & 10 min after exercise & $60.4 \pm 4.40^{w}$ & $47.7 \pm 4.2$ \\
\hline & 20 min after exercise & $65.3 \pm 4.8^{w}$ & $50.5 \pm 4.6$ \\
\hline & 30 min after exercise & $66.8 \pm 4.8^{\mathrm{tw}}$ & $53.6 \pm 4.6^{\mathrm{t}}$ \\
\hline \multirow{5}{*}{ RMSSD (ms) } & Before exercise & $60.1 \pm 5.5$ & $46.7 \pm 5.3$ \\
\hline & During exercise & $40.6 \pm 3.6^{*}$ & $39.2 \pm 3.5$ \\
\hline & $10 \mathrm{~min}$ after exercise & $66.3 \pm 5.8^{w}$ & $51.2 \pm 5.5^{\mathrm{w}}$ \\
\hline & 20 min after exercise & $70.4 \pm 6.2^{\mathrm{w}}$ & $53.3 \pm 5.9^{w}$ \\
\hline & 30 min after exercise & $70.1 \pm 6.2^{\mathrm{w}}$ & $55.2 \pm 5.9^{w}$ \\
\hline \multirow{5}{*}{ HF (n.u.) } & Before exercise & $44.7 \pm 2.6$ & $41.3 \pm 2.5$ \\
\hline & During exercise & $31.9 \pm 2.3^{*}$ & $21.2 \pm 2.2^{*}$ \\
\hline & 10 min after exercise & $47.7 \pm 2.9^{\mathrm{w}}$ & $43.8 \pm 2.8^{w}$ \\
\hline & 20 min after exercise & $46.2 \pm 3.2^{w}$ & $45.3 \pm 3.1^{w}$ \\
\hline & 30 min after exercise & $41.3 \pm 2.8^{w}$ & $40.9 \pm 2.7^{w}$ \\
\hline \multirow{5}{*}{ LF (n.u.) } & Before exercise & $50.0 \pm 2.4$ & $53.3 \pm 2.3$ \\
\hline & During exercise & $58.3 \pm 2.0 *$ & $66.7 \pm 1.9 *$ \\
\hline & 10 min after exercise & $47.9 \pm 2.7^{\mathrm{w}}$ & $50.1 \pm 2.6^{w}$ \\
\hline & 20 min after exercise & $48.9 \pm 3.0^{w}$ & $49.6 \pm 2.9^{w}$ \\
\hline & 30 min after exercise & $53.0 \pm 2.6$ & $51.0 \pm 2.5^{\mathrm{w}}$ \\
\hline \multirow{5}{*}{$\mathrm{LF} / \mathrm{HF}$} & Before exercise & $1.2 \pm 0.1$ & $1.5 \pm 0.1$ \\
\hline & During exercise & $2.2 \pm 0.4^{*}$ & $4.3 \pm 0.4^{*}$ \\
\hline & $10 \mathrm{~min}$ after exercise & $1.2 \pm 0.1^{\mathrm{w}}$ & $1.4 \pm 0.1^{\mathrm{w}}$ \\
\hline & 20 min after exercise & $1.2 \pm 0.1^{\mathrm{w}}$ & $1.4 \pm 0.1^{\mathrm{w}}$ \\
\hline & 30 min after exercise & $1.6 \pm 0.2$ & $1.5 \pm 0.2^{\mathrm{w}}$ \\
\hline
\end{tabular}

HRV - heart rate variability, SDNN - standard deviation of all normal RR intervals recorded in one-time interval, RMSSD - root mean square of the successive differences, HF - high frequency power, LF - low frequency power, LF/LH - low-to-high frequency power ratio, n.u. - normalized units

${ }^{*} p<0.05$ for before exercise vs. during exercise, ${ }^{\mathrm{t}} p<0.05$ for before exercise vs. 10, 20, and 30 min after exercise,

${ }^{\mathrm{w}} p<0.05$ for during exercise vs. 10, 20, and $30 \mathrm{~min}$ after exercise

Table 3. Post-hoc statistical power for each HRV outcome calculated from the actual correlation between repeated measures and Cohen's $f$ effect size

\begin{tabular}{lccc}
\hline HRV index & Correlation & $\begin{array}{c}\text { Effect size } \\
\text { (Cohen's } f)\end{array}$ & Power \\
\hline HF & 0.37 & 0.60 & 1.00 \\
LF & 0.29 & 0.47 & 0.99 \\
LF/HF & 0.29 & 0.68 & 1.00 \\
SDNN & 0.38 & 0.34 & 0.99 \\
RMSSD & 0.50 & 0.41 & 1.00 \\
\hline
\end{tabular}

HRV - heart rate variability, HF - high frequency power, LF - low frequency power, LF/LH - low-to-high frequency power ratio, SDNN - standard deviation of all normal RR intervals recorded in one-time interval, RMSSD - root mean square of the successive differences of stretching exercises would be determinant of autonomic modulation responses after acute training sessions. Of course, further research is needed to ratify this premise.

In our study, as expected, LF significantly increased and $\mathrm{HF}$ decreased during all exercise sessions. Along postexercise recovery, LF decreased and HF increased, reflecting a vagal compensation, compatible with the findings of prior studies [3, 7]. Consistent with these data, the LF/HF ratio increased during the exercise sessions and decreased throughout recovery. However, these responses, particularly the sympathetic withdrawal, were longer after the session with a greater vs. lower amount of exercises: 10-20 min in LA and 10-30 min in HA. In the time domain, RMSSD al- 
ways decreased during exercise and increased after exercise, irrespective of the individual's flexibility level and amount of exercises, which is in line with the premise of vagal withdrawal and re-entry, respectively [22-24]. The same pattern was observed for SDNN after the protocol including a single stretching exercise, but not after sessions with multiple exercises.

During muscle contraction or stretching, group III afferent fibres (myelinated and very sensitive to mechanical stimuli) are stimulated [17, 25], which contributes to haemodynamic adjustments mediated by the autonomic nervous system [26]. It is well accepted that increased sympathetic activity normally occurs during physical exercise, irrespective of its modality [22-24]. With regard to postexercise recovery, the initial reduction in $\mathrm{LF} / \mathrm{HF}$ was probably related to vagal re-entry [27], and later due to sympathetic lowering [23]. A basic problem of our experiment is the passivity of stretching, which probably induced lower muscle activity in comparison with exercises actively conducted by participants. However, albeit acknowledging that this fact may have reduced the stimulation of mechanoreceptors, reflex isometric contractions were still present during the exercises, especially among individuals with low flexibility levels. Our results are therefore consistent with this theoretical framework and ratify data from previous research in the sense that acute flexibility exercise can effectively produce changes in cardiac autonomic control [3-5].

Clinical trials are warranted to determine how much acute HRV changes have the potential to contribute to chronic haemodynamic adaptations, which would be of particular interest in specific clinical situations, such as in the management of patients with elevated blood pressure. In this context, the few studies that investigated long-term effects of flexibility training upon HRV are promising [5, 8]. Mueck-Weymann et al. [5] observed an increase in RMSSD ( $25.2 \pm 10.4$ vs. $62.4 \pm$ $26.9 \mathrm{~ms}, p<0.001$ ), while the LF/HF ratio decreased $(8.01 \pm 5.19$ vs. $3.44 \pm 3.53$ n.u., $p<0.02)$ after 28 days of intervention (15 min of daily stretching exercises) in healthy bodybuilders classified as having limited flexibility. Wong et al. [8] applied 8 weeks of flexibility training to obese postmenopausal women and reported reductions in $\mathrm{LF}(60.4 \pm 3.1$ vs. $46.4 \pm 2.5)$ and $\mathrm{LF} / \mathrm{HF}(1.73 \pm 0.21$ vs. $0.96 \pm 0.12)$, and increases in $\mathrm{HF}(38.5 \pm 3.1$ vs. $52.4 \pm 2.7)$ and RMSSD (3.17 \pm 0.14 vs. $3.44 \pm 0.14$ ). These findings suggest that favourable autonomic adaptations may occur owing to flexibility training, perhaps in response to a temporal summation of the effects provoked by repeated acute sessions. Albeit this possibility might be very interest- ing in terms of exercise prescription, it must be confirmed by additional research.

\section{Conclusions}

In conclusion, acute passive stretching sessions with either single or multiple exercises were capable to produce changes in HRV during exercise sessions and along postexercise recovery in individuals with low and high flexibility levels. However, the amount of exercises was determinant of the duration of these responses. During the stretching sessions, sympathetic and vagal modulations increased and decreased, respectively. On the other hand, a reduction in sympathovagal balance reflected by LF/HF occurred 20 min after the singleexercise protocol and $30 \mathrm{~min}$ after the protocol including multiple exercises. These effects were independent of the participants' flexibility level.

\section{Acknowledgements}

The authors thank Mariana Inocêncio Matos, Fábio Dutra Pereira, and Frederico de Oliveira Meirelles for the technical support. This study was supported by grants from the Brazilian Council for Scientific and Technological Development (CNPq, 309012/2010-4, recipient: Paulo Farinatti), Carlos Chagas Foundation for Research Support at Rio de Janeiro State (FAPERJ, E-26/110.184/2013, recipient: Paulo Farinatti), and Estácio de Sá University (recipient: Ercole Rubini).

\section{Disclosure statement}

No author has any financial interest or received any financial benefit from this research.

\section{Conflict of interest}

The authors state no conflict of interest.

\section{References}

1. Garber CE, Blissmer B, Deschenes MR, Franklin BA, Lamonte MJ, Lee IM, et al. American College of Sports Medicine position stand. Quantity and quality of exercise for developing and maintaining cardiorespiratory, musculoskeletal, and neuromotor fitness in apparently healthy adults: guidance for prescribing exercise. Med Sci Sports Exerc. 2011;43(7):1334-1359; doi: 10.1249/ MSS.0b013e318213fefb.

2. Rubini EC, Costa ALL, Gomes PSC. The effects of stretching on strength performance. Sports Med. 2007; 37(3):213-224; doi: 10.2165/00007256-20073703000003.

3. Farinatti PTV, Brandão C, Soares PPS, Duarte AFA. Acute effects of stretching exercise on the heart rate variability in subjects with low flexibility levels. J Strength 
Cond Res. 2011;25(6):1579-1585; doi: 10.1519/JSC. 0b013e3181e06ce1.

4. Logan JG, Yeo S. Effects of stretching exercise on heart rate variability during pregnancy. J Cardiovasc Nurs. 2017;32(2):107-111; doi: 10.1097/JCN.00000000000 00326.

5. Mueck-Weymann M, Janshoff G, Mueck H. Stretching increases heart rate variability in healthy athletes complaining about limited muscular flexibility. Clin $\mathrm{Au}-$ ton Res. 2004;14(1):15-18; doi: 10.1007/s10286-0040123-0.

6. Costa e Silva G, Conceição R, Di Masi F, Domingos T, Herdy C, Silveira A. Low intensity static stretching does not modulate heart rate variability in trained men. Medical Express. 2016;3(3):M160304; doi: 10.5935/ MedicalExpress.2016.03.04.

7. Da Silva Araujo G, Behm DG, Monteiro ER, de Melo Fiuza AGF, Gomes TM, Vianna JM, et al. Order effects of resistance and stretching exercises on heart rate variability and blood pressure in healthy adults. J Strength Cond Res. 2019;33(10):2684-2693; doi: 10.1519/JSC.0000000000002627.

8. Wong A, Sanchez-Gonzalez M, Kalfon R, Alvarez-Alvarado $S$, Figueroa $A$. The effects of stretching training on cardiac autonomic function in obese postmenopausal women. Altern Ther Health Med. 2017;23(2):20-26.

9. Dong J-G. The role of heart rate variability in sports physiology (review). Exp Ther Med. 2016;11(5):15311536; doi: 10.3892/etm.2016.3104.

10. Goldberger JJ, Le FK, Lahiri M, Kannankeril PJ, Ng J, Kadish AH. Assessment of parasympathetic reactivation after exercise. Am J Physiol Heart Circ Physiol. 2006; 290(6):H2446-H2452; doi: 10.1152/ajpheart.01118. 2005.

11. Ng J, Sundaram S, Kadish AH, Goldberger JJ. Autonomic effects on the spectral analysis of heart rate variability after exercise. Am J Physiol Heart Circ Physiol. 2009;297(4):H1421-H1428; doi: 10.1152/ajpheart.00217.2009.

12. Cottin F, Médigue C, Papelier Y. Effect of heavy exercise on spectral baroreflex sensitivity, heart rate, and blood pressure variability in well-trained humans. Am J Physiol Heart Circ Physiol. 2008;295(3):H1150-H1155; doi: 10.1152/ajpheart.00003.2008.

13. Perini R, Veicsteinas A. Heart rate variability and autonomic activity at rest and during exercise in various physiological conditions. Eur J App Physiol. 2003; 90(3-4):317-325; doi: 10.1007/s00421-003-0953-9.

14. Huang P-H, Leu H-B, Chen J-W, Lin S-J. Heart rate recovery after exercise and endothelial function - two important factors to predict cardiovascular events. Prev Cardiol. 2005;8(3):167-170; doi: 10.1111/j.1520-037x. 2005.3847.x.

15. Tereshchenko LG, Cygankiewicz I, McNitt S, Vazquez R, Bayes-Genis A, Han L, et al. Predictive value of beatto-beat QT variability index across the continuum of left ventricular dysfunction: competing risks of non- cardiac or cardiovascular death and sudden or nonsudden cardiac death. Circ Arrhythm Electrophysiol. 2012;5(4):719-727; doi: 10.1161/CIRCEP.112.970541.

16. Khandoker AH, Jelinek HF, Palaniswami M. Identifying diabetic patients with cardiac autonomic neuropathy by heart rate complexity analysis. Biomed Eng Online. 2009;8:3; doi: 10.1186/1475-925X-8-3.

17. Gladwell VF, Coote JH. Heart rate at the onset of muscle contraction and during passive muscle stretch in humans: a role for mechanoreceptors. J Physiol. 2002; 540(Pt 3):1095-1102; doi: 10.1113/jphysiol.2001.013486.

18. Murata J, Matsukawa K. Cardiac vagal and sympathetic efferent discharges are differentially modified by stretch of skeletal muscle. Am J Physiol Heart Circ Physiol. 2001;280(1):H237-H245; doi: 10.1152/ajpheart.2001. 280.1.H237.

19. De Araújo CGS. Flexitest - an innovative flexibility assessment method. Champaign: Human Kinetics; 2004.

20. De Araújo CGS. Flexibility assessment: normative values for Flexitest from 5 to 91 years of age. Arq Bras Cardiol. 2008;90(4):257-263; doi: 10.1590/s0066-782x 2008000400008.

21. Task Force of the European Society of Cardiology and the North American Society of Pacing and Electrophysiology. Heart rate variability: standards of measurement, physiological interpretation, and clinical use. Circulation. 1996;93(5):1043-1065; doi: 10.1161/01.CIR.93. 5.1043.

22. Wilson LB, Wall PT, Pawelczyk JA, Matsukawa K. Cardiorespiratory and phrenic nerve responses to graded muscle stretch in anesthetized cats. Respir Physiol. 1994; 98(3):251-266; doi: 10.1016/0034-5687(94)90075-2.

23. Gallo Júnior L, Maciel BC, Marin-Neto JA, Martins LE. Sympathetic and parasympathetic changes in heart rate control during dynamic exercise induced by endurance training in man. Braz J Med Biol Res. 1989;22(5):631643.

24. Stebbins CL, Brown B, Levin D, Longhurst JC. Reflex effect of skeletal muscle mechanoreceptor stimulation on the cardiovascular system. J Appl Physiol. 1988;65(4): 1539-1547; doi: 10.1152/jappl.1988.65.4.1539.

25. Kaufman MP, Longhurst JC, Rybicki KJ, Wallach JH, Mitchell JH. Effects of static muscular contraction on impulse activity of groups III and IV afferents in cats. J Appl Physiol Respir Environ Exerc Physiol. 1983; 55(1 Pt 1):105-112; doi: 10.1152/jappl.1983.55.1.105.

26. Matsukawa K, Wall PT, Wilson LB, Mitchell JH. Reflex stimulation of cardiac sympathetic nerve activity during static muscle contraction in cats. Am J Physiol Heart Circ Physiol. 1994;267(2 Pt 2):H821-H827; doi: 10.1152/ajpheart.1994.267.2.H821.

27. Imai K, Sato H, Hori M, Kusuoka H, Ozaki H, Yokoyama $\mathrm{H}$, et al. Vagally mediated heart rate recovery after exercise is accelerated in athletes but blunted in patients with chronic heart failure. J Am Coll Cardiol. 1994; 24(6):1529-1535; doi: 10.1016/0735-1097(94)90150-3. 